\title{
Is a Picture Worth a Thousand Words? Evidence from Concept Definitions by Patients with
} Semantic Dementia

\author{
Matthew A. Lambon Ralph, Kim S. Graham, and Karalyn Patterson \\ MRC Cognition and Brain Sciences Unit, Cambridge, United Kingdom
}

and

John R. Hodges

\begin{abstract}
MRC Cognition and Brain Sciences Unit, Cambridge, United Kingdom; and University Neurology Unit, Addenbrooke's Hospital, Cambridge, United Kingdom
\end{abstract}

Nine patients with semantic dementia (the temporal lobe variant of frontotemporal dementia) were asked to define concrete concepts either from presentation of a picture of the object or from its spoken name. As expected, the patients with the most severe semantic impairment produced the least detailed definitions, and the quality of the definitions overall was significantly related to concept familiarity. Further analyses of the definitions were designed to assess two key theoretical aspects of semantic organization. (i) Do objects and their corresponding names activate conceptual information in two neuroanatomically separable (modality-specific) semantic systems? If so, then-apart from any expected commonality in performance attributable to factors such as concept familiarity-one would not predict striking item-specific similarities in a patient's picture- and word-elicited definitions. (ii) Do sensory/perceptual features and more associative/functional attributes of conceptual knowledge form two neuroanatomically separable subsystems? If so, then one would predict significant dissociations in the prominence of these two types of information in the patients' definitions. The results lead us to favor a model of the semantic system that is divided by attribute type but not by modality. (c) 1999 Academic Press

We are indebted to the continuing participation of the patients included in this paper; to Jim Becker, Kellie Doyle, and Steve DeKoskey (University of Pittsburgh) for allowing us to report GC's data; and to Marge Eldridge for her assistance with scoring the patients' definitions. The research was supported by funding from the National Institutes of Health (USA) and the Medical Research Council (UK). Address correspondence and reprint requests to Dr. M. A. Lambon Ralph, MRC Cognition and Brain Sciences Unit, 15 Chaucer Road, Cambridge CB1 2EF, United Kingdom. E-mail: matt.lambon.ralph@msc-cbu.cam.ac.uk. 


\section{INTRODUCTION}

Semantic dementia or progressive fluent aphasia arises from a neurodegenerative disease that, at least in early to moderate stages, is associated with highly circumscribed atrophy of the inferior and lateral aspects of the temporal neocortex (Hodges \& Patterson, 1996; Hodges, Patterson, Oxbury, \& Funnell, 1992; Mummery, Patterson, Wise, Price, \& Hodges, 1999; Snowden, Goulding, \& Neary, 1989). The cognitive decline in this form of frontotemporal dementia is characterized by a progressive loss of conceptual knowledge leading to poor comprehension and pronounced word-finding difficulties. Performance on tasks that tap other aspects of perceptual and cognitive processing - for example, those that assess day-to-day memory, syntax, single word phonology, spatial abilities, and nonverbal reasoning-remains relatively intact (Hodges et al., 1992; Hodges, Patterson, \& Tyler, 1994). As noted initially by Warrington (1975) and Schwartz, Marin, and Saffran (1979), the selective impairment of semantic memory in these patients allows researchers to observe the nature and consequences of degraded conceptual knowledge without the cooccurring symptoms in other disorders that also lead to semantic impairment, such as dementia of Alzheimer's type (DAT) or herpes simplex virus encephalitis (HSVE).

In this study nine patients with semantic dementia were asked to provide definitions of 48 concepts, presented (on separate occasions) as both pictures and spoken names of the concepts. The analyses of these definitions were designed to assess two theoretical aspects of semantic organization: whether patterns of selective impairment require theorists to postulate (i) separate conceptual representations accessed by objects (pictures) and words and (ii) separate subsets of semantic attributes differentiated by feature type. Note that by separable subsystems we mean two component processes that need not be completely independent when functioning normally, but nevertheless have sufficiently separate neuroanatomical bases as to be selectively disruptable by circumscribed lesions (Shallice, 1988a). For example, as we shall argue later, sensory/perceptual and associative/functional attributes may be underpinned by different neuroanatomical regions and therefore differentially vulnerable to lesion. Under normal circumstances, however, these separate brain regions are mutually active and interactive, giving rise to the multifaceted representations of semantic memory.

\section{Modality-Specific Semantic Organization?}

Some theorists have argued for separate sets of conceptual representations associated with different input modalities (McCarthy \& Warrington, 1986; McCarthy \& Warrington, 1988; Shallice, 1988b; Shallice, 1993; Warrington \& McCarthy, 1994). We should note at the outset that, by modality, these theorists mean not sensory systems of input (vision, hearing, touch) but rather objects (or pictures of them) versus words (spoken or written). 
According to this view, it should be possible to identify patients who form a double dissociation between picture and word comprehension. At least a single dissociation in this regard seems well established, as a number of patients have been documented with poor comprehension of words relative to pictures (e.g., Bub, Black, Hampson, \& Kertesz, 1988; Graham, Becker, Patterson, \& Hodges, 1997a; Lambon Ralph, Howard, Nightingale, \& Ellis, 1998b; Lauro-Grotto, Piccini, \& Shallice, 1997; McCarthy \& Warrington, 1988; Tanabe, Nakagawa, Ikeda, Hashimoto, Yamada, Kazui, Nishikawa, \& Okuda, 1996).

The opposite pattern, impaired visual semantics but intact verbal comprehension, has also been reported. For example McCarthy and Warrington (1986) described patient FRA who was able to define spoken words but could not mime or name some of the same items when presented as pictures. McCarthy and Warrington argued for a semantic locus for this effect on two grounds. First, FRA made semantic errors in naming/defining pictures; second, the patient demonstrated a category-specific advantage for the naming of actions (83\%) over objects (50\%). In another case described by Warrington and McCarthy (1994), patient DRS was error-free (15/15) in naming from description but had a degree of anomia for object pictures (Graded Naming Test-11/30: McKenna \& Warrington, 1980). Although he failed to show a category-specific deficit on naming colored photographs of manmade objects and animals, DRS exhibited significantly worse performance for common objects than for animals when required to match the same item across two different views (visual-visual matching) or in word-to-picture matching. Given the combination of a category-specific pattern and a specific deficit in the recognition of visually presented items, Warrington and McCarthy concluded that DRS's performance arose from a selective impairment of visual semantics.

In contrast to the multiple semantics hypothesis, other researchers have proposed theories in which stimuli from each modality access the same set of conceptual representations (Caramazza, Hillis, Rapp, \& Romani, 1990; Hillis, Rapp, \& Caramazza, 1995; Lambon Ralph, Patterson, \& Hodges, 1997; Rapp, Hillis, \& Caramazza, 1993; Riddoch, Humphreys, Coltheart, \& Funnell, 1988). In a functional imaging study with normal subjects, Vandenberghe, Price, Wise, Josephs, and Frackowiak (1996) identified a large semantic network activated in common by both words and pictures that extended from the left superior occipital gyrus through the middle and inferior temporal cortex to the inferior frontal gyrus. In studies of semantic memory in patients with DAT, Hodges, Patterson, Graham, and Dawson (1996) and Lambon Ralph et al. (1997) found a clear relationship between the richness of semantic attributes given in definitions to concept names and the patients' success on the same items when presented as pictures for naming to confrontation. If the subsets of knowledge accessed by pictures and words are represented in separate systems, there is no reason to expect a reliable associa- 
tion across these two tasks in patients with a central semantic deficit. In a similar study of the semantic impairment in DAT, Chertkow, Bub, and Caplan (1992) also observed a strong association between performance on probe questions whether presented with a picture or the corresponding name.

The item-by-item association reported in neuropsychological studies by Chertkow et al. (1992), Hodges et al. (1996), and Lambon Ralph et al. (1997), plus the common neural substrate for word and picture comprehension highlighted by the functional imaging study of Vandenberghe et al. (1996), seems to conflict with the apparent evidence for a double dissociation between visual and verbal semantic systems. We suggest, however, that there might be alternative explanations for the evidence presented in favor of the modalityspecific semantics view. For example, it is possible that FRA (McCarthy \& Warrington, 1986) and/or DRS (Warrington \& McCarthy, 1994) had a subtle perceptual deficit that might have contributed to the apparent disadvantage for visual comprehension. Although these patients succeeded on some perceptual assessments that required no verbal output, both had lesions to occipital or occipito-temporal regions, were completely alexic, and demonstrated better naming in response to a verbal description of an object than to a picture of it. Warrington and McCarthy argued for a semantic locus in these two cases, and thus for the existence of a separate visual semantic system, because performance in each was affected by semantic category and because FRA's predominant error type was semantic. There are, however, other possible interpretations of the category-specific pattern. For example, a mild visual perceptual deficit and a mild category-specific deficit at the conceptual level could combine to form an apparent category-specific deficit in the visual domain. Alternatively, the category-specific deficits may relate in part to factors such as familiarity that can yield differential levels of difficulty for the two domains. Furthermore, with regard to the semantic errors observed in patient FRA, it is possible that a pre-semantic visual impairment might engender this type of error. In fact it is clear from implemented computational models that error type and locus of impairment do not have a one-to-one relationship (cf. Plaut \& Shallice, 1993).

Without additional evidence for a selective impairment of visual semantics, one-half of the double dissociation that underpins the modality-specific view remains in doubt. If we turn our attention to the opposite pattern, we could ask how do the amodal semantic theories account for the patients with disproportionately poor verbal comprehension? Many theorists have emphasized the underlying difference between words and objects in terms of their relationship with conceptual knowledge: the surface form of a word, unlike an object or picture, has an arbitrary relationship with its meaning. Morton (p. 223, 1985) noted that "if we recognize part of an object we can often say a great deal about what the object is" and invoked Gibson's (1979) notion of affordance to refer to those properties of the whole object that can 
be determined without full recognition of its specific identity. Affordance seems similar in many ways to the processing assumptions adopted by Caramazza and colleagues (Caramazza et al., 1990; Hillis et al., 1995). They suggest that object stimuli, but not words, allow direct access to meaning (the assumption of privileged access) and that, because the relationship between form and function is not arbitrary, knowledge about the structural aspects of an object will be closely linked to the semantic properties that specify function (the assumption of privileged relationships). These two assumptions taken together imply that not only structural but also functional aspects of concept knowledge should be more readily accessed from the object (or a picture of it) than from its name.

In addition to the notion of affordance, a number of additional observations are worthy of consideration with regard to the patients reported to have poorer comprehension of words than pictures. Graham et al. (1997a) analyzed the definitions given by a patient with semantic dementia (GC) to a set of concepts presented as either pictures or words. Although GC did produce better definitions in response to picture stimuli, Graham et al. argued that this extra information was largely attributable to the features that were visible in, or inferable from, the picture itself. Furthermore, GC's definitions for picture stimuli (and comprehension assessed in other object-based tasks such as the Pyramids and Palm Trees test: Howard \& Patterson, 1992) were not normal either. Other patients with selectively impaired verbal comprehension have had measurable deficits in picture comprehension, too, when this has been assessed in some detail. For example, the patient RM described by Lauro-Grotto et al. (1997) performed at chance when required to sort pictures at the level of subordinate features and was extremely impaired on the picture as well as the word version of the Pyramids and Palm Trees test. Although she exhibited a large advantage in demonstrating the appropriate cooking procedure for the real item relative to its name, she provided little or no information in response to a number of the foodstuffs, including two that, presumably, were premorbidly very familiar to this Italian subject (aubergine and gnocchi).

In summary, it is not clear that there is compelling evidence for the modality-specific view of semantic organization. One of the lessons to be learned from connectionist models is that the underlying relationship between two sets of representations has a major impact on behavior. Performance is more robust to the effects of damage if the input and output representations have a principled relationship (quasi-regular systems, cf. Plaut, McClelland, Seidenberg, \& Patterson, 1996). Within this framework verbal comprehension suffers from the arbitrary relationship between the surface form (phonology/orthography) and meaning. For pictures, however, there is a degree of systematicity in the mapping from the surface form to meaning and it is this systematicity that leads to the affordances enjoyed by object comprehension. 


\section{Selective Impairment to a Subset of Semantic Attributes}

A number of theorists have proposed that conceptual representations are made up from a distributed pattern of sensory and associative/functional attributes (e.g., Allport, 1985; Lambon Ralph et al., 1997; Saffran \& Schwartz, 1994; Saffran \& Sholl, in press; Warrington \& McCarthy, 1987). These different types of attribute will contribute, in general, to each and every conceptual representation, but the relative weighting will vary across objects and possibly across different semantic categories. If we assume that, because they are linked to different sources of input and are learned in different ways, these types of attribute are underpinned by different brain regions, then it should be possible to find patients for whom certain types of knowledge have been selectively compromised. Relatively impaired visuo-perceptual information has been reported in a number of patients (e.g., Basso, Capitani, \& Laiacona, 1988; Breedin, Saffran, \& Coslett, 1994b; Carbonnel, Charnallet, David, \& Pellat, 1997; Cardebat, Demonet, Celsis, \& Puel, 1996; Coltheart, Inglis, Cupples, Michie, Bates, \& Budd, 1998; Lambon Ralph et al., 1998b; Marshall, Pring, Chiat, \& Robson, 1996; Moss, Tyler, Hodges, \& Patterson, 1995; Sirigu, Duhamel, \& Poncet, 1991; Srinivas, Breedin, Coslett, \& Saffran, 1997; Tyler \& Moss, 1997).

The possibility that it is particularly knowledge of the visuo-perceptual features of concepts that is degraded in semantic dementia takes on special interest given that the disorder is associated with progressive atrophy of the inferolateral temporal lobe (Hodges et al., 1992; Mummery et al., 1999) a region that is commonly linked to high-order visual processing and object recognition (Milner \& Goodale, 1995; Ungerleider \& Mishkin, 1982). Although not the main focus of many existing papers in the literature, poor knowledge of sensory/visual attributes has been noted in a number of studies of semantic dementia. For example, patient DM (Breedin, Martin, \& Saffran, 1994b; Srinivas et al., 1997) was less successful at answering perceptual questions about animals and objects (perceptual questions, 69\%; nonperceptual questions, 86\%). Furthermore, although he achieved respectable performance on many aspects of verb knowledge, he was poor at matching the meaning of verbs on the basis of manner for which, it is argued, sensorimotor features are critical. Tyler and her colleagues (Moss et al., 1995; Tyler \& Moss, 1997, 1998) have reported several patients with significant semantic priming based on a functional relationship between prime and target (e.g., fox-sly) but either no priming or reduced priming with regard to perceptual features (e.g., fox-red). The patient reported by Lambon Ralph et al. (1998b) was less likely to match a definition to the name of the correct concept if the definition contained perceptual rather than functional/associative information, was significantly worse at answering questions about sensory than nonsensory attributes, and produced significantly less perceptual than nonperceptual information in her definitions of words. 
In this case-series study we report the analyses of definitions given by nine patients with semantic dementia for a set of 48 different concepts. Patients were asked to give definitions (on different occasions) both to a picture and to the spoken name of each concept. The results are reported in two main sections designed to address the aspects of semantic organization noted above; namely, does the conceptual knowledge accessed by words and pictures form two separable components of the semantic system, and do visual/ sensory and associative features of conceptual knowledge form two separable components of the semantic system?

\section{CASE REPORTS}

Some of the nine patients tested in this study have been described in previous papers (Graham \& Hodges, 1997; Graham, Hodges, \& Patterson, 1994; Graham, Lambon Ralph, \& Hodges, 1997b; Graham, Murre, \& Hodges, in press; Hodges \& Graham, 1998; Knott, Patterson, \& Hodges, 1997; Lambon Ralph, Graham, Ellis, \& Hodges, 1998a; Mummery et al., 1999; Tyler \& Moss, 1997, 1998), including an analysis of the definition data included here for one of the patients (GC, Graham et al., 1997a). Table 1 shows background neuropsychological and neuroanatomical information for the nine patients at the time that definitions of pictures and words were collected. Where available, scores for a group of 24 age-matched control subjects are also included in Table 1 (mainly from Hodges \& Patterson, 1995). In Table 1 and the subsequent figures, the patients are ordered first by the relative laterality of their temporal lobe damage (see below) and then by comprehension (as measured by word-picture matching) with the least impaired patient on the left of each group.

Although there was evidence of bilateral involvement in all nine cases, assessment of coronal T1-weighted MRI sections revealed predominantly left-sided temporal lobe atrophy in seven cases (JC through AM), with greater atrophy of the right temporal lobe in the remaining two patients (BM and DG). Most of the patients produced a good copy of the Rey Figure (Osterrieth, 1944) and some recalled as much after a 45-min delay as control subjects do (as the standard deviation on normal performance suggests, intact subjects' performance varies a great deal). Forward and backward digit span was within the normal range for all nine patients. Nonverbal problem solving as assessed by Raven's Coloured Progressive Matrices (Raven, 1962) was equal to or better than the age-appropriate norm in the patients tested.

The patients' semantic impairments and profound anomia were highlighted by a series of assessments. Fluency was reduced in all nine patients for eight different semantic categories (four living categories: land animals, birds, water creatures, and dogs; and four man-made classes: household items, vehicles, musical instruments, and boats). All patients were anomic, some profoundly so, as measured by naming of a set of 48 simple line draw- 


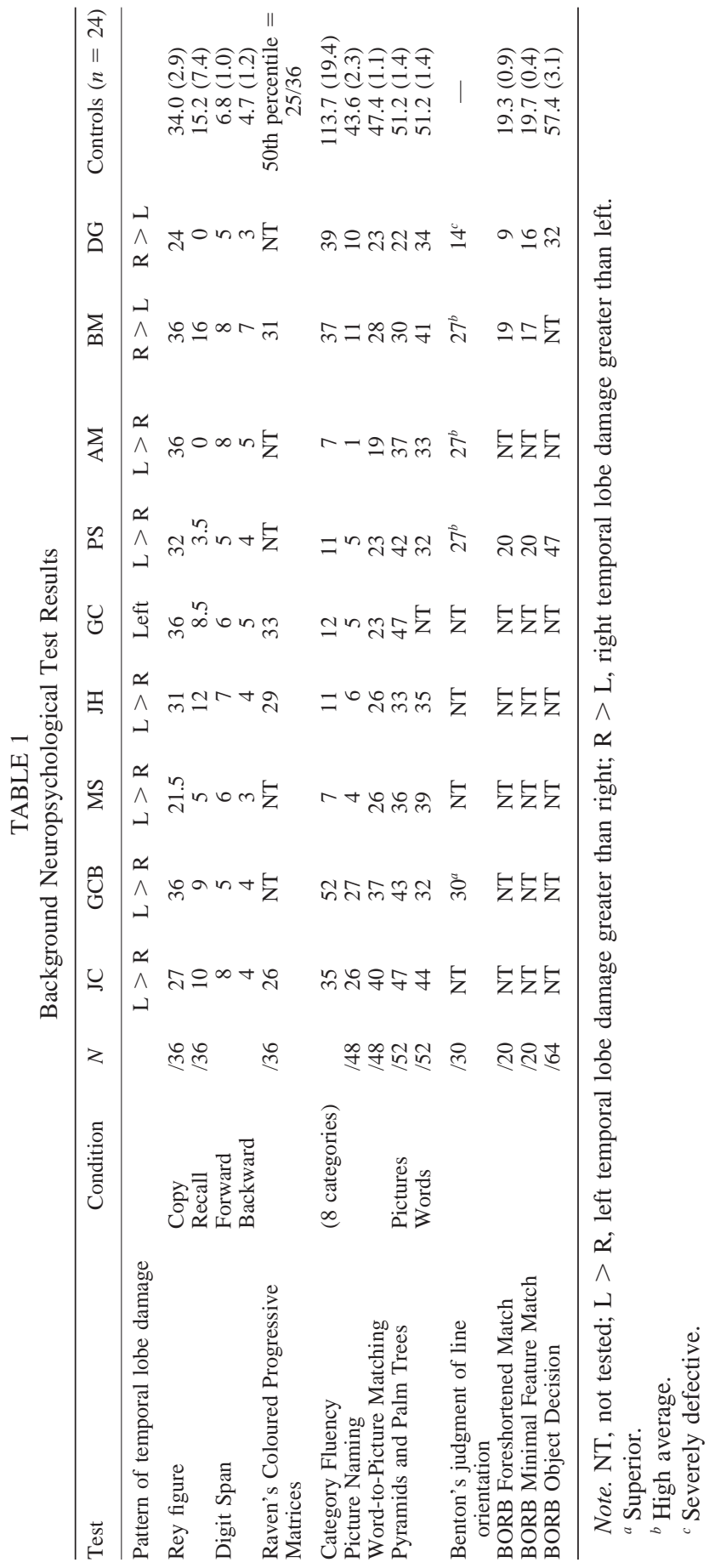


ings of common objects (24 animals and 24 artifacts drawn from Snodgrass \& Vanderwart, 1980) and all performed outside the normal range when asked to match the spoken name to the corresponding picture for the same set of items (in this word-to-picture matching test the target is presented with seven other items from the same semantic category). Their poor comprehension was confirmed by performance on the Pyramids and Palm Trees test (Howard \& Patterson, 1992). Two observations about this latter assessment are relevant to the current study. First, all nine patients were outside the normal range for both picture and word versions confirming impaired comprehension for both input modalities. Second, relative performance for pictures and words seems to align with the pattern of temporal lobe damage: the patients with predominantly left-sided atrophy produced either similar scores for the two versions or better performance for pictures over words; the two patients with relatively right-sided temporal lobe atrophy (BM and DG), however, achieved higher scores for words than for pictures. Indeed neither BM nor DG exceeded chance level (50\%) with pictures.

At the time that the definitions were collected, we did not systematically investigate perceptual functioning in these patients. The limited results available are shown at the bottom of Table 1 . Four of the five patients assessed performed at the high average or superior range on Benton's Judgement of Line Orientation (Benton, Hamsher, Varney, \& Spreen, 1983), but DG's score classifies as severely defective. On three tasks taken from the Birmingham Object Recognition Battery (BORB: Riddoch \& Humphreys, 1992), PS performed at ceiling on the two matching tests but outside the normal range for object decision. Patient $\mathrm{BM}$ was within the normal range for the Foreshortened Match assessment but fell outside the normal range on the simple Minimal Feature Match test. DG's poor perceptual processing was confirmed with these tasks: she performed at chance on the Foreshortened Match and Object Decision tests and was outside the normal range on the Minimal Feature Match assessment. It is interesting to note at this point, prior to consideration of their definitions to pictures and words, that BM's and DG's better performance on the Pyramid and Palm Trees test with words than pictures coincided with poor matching of objects across different views.

Definitions for the same 48 items used in the word-to-picture matching and naming tasks (reported in Table 1) were collected from each patient. On one occasion each patient was asked to define the 48 items presented as Snodgrass and Vanderwart (1980) line drawings, and on the other they were asked to define the spoken name of each concept. On both occasions the patients were encouraged to provide as rich a definition as possible, as though the listener was completely unfamiliar with all the items. Following the guidelines described in Hodges et al. (1996), each definition was scored for the following information: whether the information provided was sufficient to define the core meaning of the object, that is, contained enough informa- 
tion for another person to identify the concept from the definition; the total number of correct features produced with regard to physical/sensory attributes (e.g., a table has four legs, an ostrich has a long neck, etc.) and of an associative/functional nature (e.g., a table is used for eating at, ostriches live in Africa, etc.); and the presence or absence of a superordinate category label. An attribute was classified as physical/sensory if it referred to any physical feature that a person might know about an object, whether or not it can be perceived in a simple line drawing. The classification of associative/functional was used for all nonphysical information, encompassing knowledge about an object's function, information that is associated with an item, and any encyclopedic facts. For definitions of pictures the number of physical attributes was further subdivided into the number of physical features that are included in the picture itself versus the number of novel physical attributes mentioned. When a specific feature was repeated in a definition, the attribute was only counted once; i.e., the value reported for each type of feature reflects the type count, and not a token count.

We should note here that, for the sake of objective scoring, each definition was taken at face value-i.e., only those attributes mentioned by each patient were included. Additional knowledge that might be inferred from the definition was not credited. So, for example, when one patient (DG) initially responded "Is that a fish?" to a picture of a crocodile, her scores for that item were not credited with additional features such as that she considered the target picture to represent a water creature. Our experience with these patients, both clinical and experimental, suggests that it is very easy to overestimate the degree of remaining comprehension by making this kind of inference from the patient's responses. In fact, the conservative scoring scheme adopted here was justified by DG's continuing definition of the crocodile in that she subsequently commented, "Ain't a dog, is it?"

\section{Preliminary Analyses}

Despite only having nine patients within this case series, we found a significant relationship between the quality of the patients' definitions and the degree of their semantic impairment: that is, cross-sectionally, the total amount of information produced to words and pictures declined as comprehension decreased (correlation between the patients' word-to-picture matching score and the total number of novel features produced for pictures: $r=$ $.66, P_{\text {one-tailed }}=.03$; correlation between the patients' word-to-picture matching score and the total number of features produced for words: $r=.63$, $P_{\text {one-tailed }}=.03$ ). The familiarity of the concept also significantly affected the patients' performance: the total number of features produced to words and pictures declined as the concepts became relatively less familiar (correlation between rated familiarity of each item and the number of features produced per word: $r=.37, P_{\text {one-tailed }}<.001$; correlation between rated familiarity of 
TABLE 2

The Relationship between Core Definitions Given to Pictures and to Their Names

\begin{tabular}{lccc}
\hline & \multicolumn{2}{c}{$\begin{array}{c}\text { Core word } \\
\text { definition }\end{array}$} \\
\hline & & $\boldsymbol{*}$ & $\checkmark$ \\
Core picture definition & $\boldsymbol{*}$ & 247 & 41 \\
& & 55 & 89 \\
\hline
\end{tabular}

Note. $C=.44, p<.001$.

each item and the number of novel features produced per picture: $r=.20$, $\left.P_{\text {one-tailed }}<.001\right)$. These two preliminary results confirm (a) that definitions are a sensitive measure of the deterioration of conceptual knowledge characteristic of semantic dementia (Warrington, 1975) and (b) that this deterioration is modulated by the frequency or familiarity of the concepts assessed, with less familiar items more vulnerable (Funnell, 1995).

\section{Modality-Specific Semantic Impairments?}

Table 2 shows the distribution of definitions for pictures and words collected from all nine patients, where each definition was scored for the presence of sufficient information to be categorized as a core definition. There was a clear and significant concordance between performance on the same items presented as words and pictures $(C=.44, p<.001)$. As the patients had been asked, on a separate occasion, to name each of the 48 pictures (see Table 1), we were also able to compare naming accuracy against definitions to words. If the information produced was scored to the core definition criterion, there was also a significant item-specific concordance between picture naming and definitions of concept names (see Table 3, $C=.50, p<.001$ ).

We have already demonstrated that the quality of the patients' definitions was influenced by item familiarity, a fact that would-in and of itself-

TABLE 3

The Relationship between Picture Naming Accuracy and Core Definitions

to Picture Names

\begin{tabular}{lccc}
\hline & \multicolumn{2}{c}{$\begin{array}{c}\text { Core word } \\
\text { definition }\end{array}$} \\
\hline & \multirow{*}{*}{$\begin{array}{c} \\
\text { Picture naming }\end{array}$} & 283 & 54 \\
\hline
\end{tabular}

Note. $C=.50, p<.001$. 
yield some consistency (cf. Behrmann \& Bub, 1992; Coltheart \& Funnell, 1987; Howard, 1995). That is, some items will always be hard, because of low familiarity (and any other item-specific properties affecting difficulty), and thus engender poor definitions and low naming scores. Other items will be easy and so produce consistently better performance. This alternative explanation for the item-specific associations was assessed via two simultaneous logistic regression analyses. Picture naming accuracy was predicted by word definition performance, and word definition by picture definition. In both analyses rated concept familiarity and a range of other psycholinguistic factors that might have influenced performance were included in the regression equation (name length in phonemes; combined frequency per million from Baayen, Piepenbrock, \& van Rijn, 1993; and rated visual complexity, imageability, age-of-acquisition, and familiarity from Coltheart, 1981). Word definition performance was found to be a significant predictor of naming accuracy even after the influence of the six factors was taken into account (Wald $=55.9, p<.0001$ ). Likewise, success in picture definition was a significant predictor of word definition accuracy (Wald $=50.2, p<.0001$ ) with the influence of the other six factors removed.

In the Introduction we noted two results from studies of patients with DAT — a significant association between picture naming success and quality of definitions to the picture names (Lambon Ralph et al., 1997) and a strong association between performance on probe questions whether presented with a picture or the corresponding name (Chertkow et al., 1992). These patterns are replicated by our data from nine patients with semantic dementia, where the association between quality of concept definition to word and picture, and also between picture naming and word definition, was significant over and above the influence of item familiarity and other variables. These reliable associations are a clear prediction from the hypothesis of an amodal semantic system that is addressed by both word and picture input.

The patients' definitions were compared further by studying the number of attributes produced to pictures and words, illustrated in Fig. 1. Two numbers are given for picture definitions. The first (Picture: Total) represents the total number of attributes produced, both associative and physical; the second (Picture: Novel) includes associative features and only those perceptual physical features that are not contained within the stimulus picture itself. Thus the measure Picture: Novel is a more conservative estimate of what a subject knows about a pictured concept rather than of what he or she can find in the stimulus.

Graham et al. (1997a) noted that patient GC tended to produce information in picture definitions that was visible in the drawing itself. This tendency can also be seen for a few of the cases in Fig. 1, as the difference between the total number of features produced in response to pictures and the number of novel attributes given (Picture: Total vs. Picture: Novel). This figure also demonstrates that, despite their impoverished semantic knowledge, the ma- 


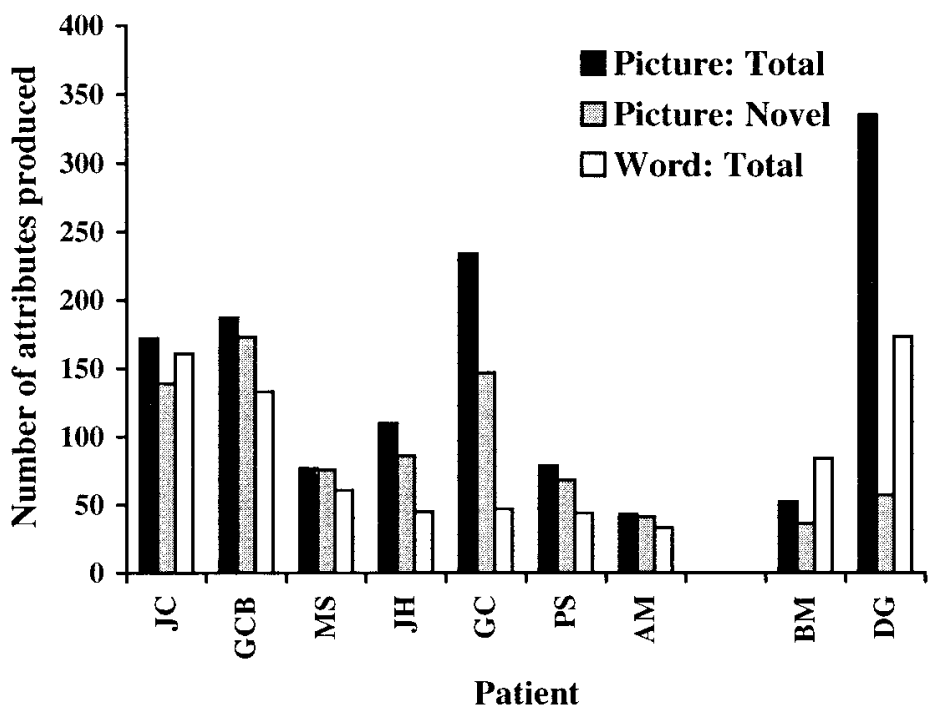

FIG. 1. The number of attributes produced to pictures and words. Picture: Total-the total number of attributes produced to pictures. Picture: Novel-the total number of associative features and only those perceptual physical features that are not contained within the stimulus picture itself. Word: Total— the total number of attributes produced to words.

jority of the patients did not resort to this kind of simple listing of the details contained in the pictures. The clear exception to this, apart from GC himself, was DG who produced 335 separate pieces of information to the 48 line drawings, of which 278 features (83\%) were present in the picture. Returning to DG's definition of a crocodile as an example, her full response was, "Is that a fish? I don't know what it is? Got a head, four legs and a tail. Ain't a dog is it? A bit flat, tail, four legs, head, two eyes, mouth, toes, several toes.' DG's apparent strategy to list components of the item pictured is important for the comparison between word and picture definitions: based on total number of features produced, this comparison would suggest that DG provided more information to pictures than to words; but if only the novel physical information is counted, then DG's performance was, in fact, substantially worse for pictures than for words.

As a group, the patients with relatively greater left temporal lobe damage produced significantly more correct information in response to pictures (novel physical plus associative) than to words (Wilcoxon $Z=5.45, p<$ $.001)$. The reverse was true for the two patients with greater right temporal lobe atrophy (combined data: Wilcoxon $Z=5.97, P<.001$ ). If each patient's data are considered individually, then the advantage for pictures over words was significant in four of the patients with left-sided atrophy (by Wilcoxon, GCB: $Z=2.15, p=.03$; JH: $Z=3.49, p<.001$; GC: $Z=5.41$, $p<.001$; PS: $Z=2.34, p=.02$ ) but did not reach significance in the 
remaining three individuals (JC, for whom in fact the nonsignificant difference was in the opposite direction, MS, and AM). Both patients with relatively right-sided temporal atrophy produced significantly richer definitions to words than to pictures (BM: $Z=3.77, p<.001$; DG: $Z=4.62, p<$ $.001)$.

The notion of an inherent difference between pictures and words in the nature of their relationship to conceptual attributes-principled in the case of pictures, arbitrary for words-leads to the following prediction. When semantic knowledge is degraded, patients should still often be able to say something appropriate about concepts presented as pictures, at least with regard to properties of the correct semantic category, whereas for words there should be a much greater proportion of occasions on which the patient is unable to say anything appropriate about the concept. This prediction was supported by the patients' definition data. Combining across all nine patients the number of items for which absolutely no appropriate attributes were produced was much greater for words ( $46 \%$ of the items) than for the corresponding pictures, whether the picture definitions were scored to include all the information produced (18\% of the items: a significant difference: Sign test, $p<.001)$ or to disregard description of the physical information included within the picture $(27 \%$ of the items: Sign test, $p<.001)$. If each patient's data are considered individually, then for six of the patients (JC, $\mathrm{GCB}, \mathrm{JH}, \mathrm{GC}, \mathrm{PS}$, and DG) the number of items for which absolutely no appropriate attributes were produced was significantly greater for words (the proportion for individual patients ranged from 19 to $77 \%$ of the concepts) than for the corresponding pictures scored to include all the information produced (the proportions ranged from 0 to 23\%: individual patient Sign tests, $p$ values ranged from $p<.001$ to $p=.02)$. The difference failed to reach significance for three patients (AM, MS, and BM). With one exception, the pattern for each patient was unchanged if the Picture: Novel data were substituted for Picture: Total. The exception was DG who failed to produce any appropriate attributes for $33 \%$ of the words. If her picture definitions are scored by the lenient criterion, then she produced some information for every picture (a significant difference: Sign test, $p<.001$ ) but if the physical information included within the stimulus picture itself is discounted there is no significant difference (DG failed to produce any novel information for $38 \%$ of the pictures).

In summary of this aspect of the results, there was an impressive degree of item-specific consistency between the patients' definitions to pictures and words (and also between their definitions and naming to confrontation) that could not be attributed to factors such as concept familiarity. Furthermore, although some of the patients achieved significantly better definitions to either pictures or words (relative to the other modality), we claim that these differences can be explained without recourse to modality specific semantic subsystems. In particular, (a) the superiority in picture definitions is almost 
certainly explicable in terms of information afforded by picture stimuli, and (b) the less frequently observed advantage for words is most likely to reflect a presemantic visuo-perceptual deficit cooccurring with degraded conceptual knowledge. These views are expanded in the General Discussion.

\section{Selective Impairment to a Subset of Semantic Attributes?}

Figures $2 \mathrm{a}$ and $2 \mathrm{~b}$ illustrate how the total number of features produced by each patient divides into associative and physical attributes, for picture and word definitions respectively. ${ }^{1}$ In nearly all cases the overwhelming proportion of information provided, for both picture and word stimuli, was associative. In some cases the amount of physical information produced was extremely small (all nine patients, on average, produced less than one novel physical attribute per item for pictures, and the same is true for seven of the nine patients in the word definitions; recall that the values in Fig. 2 are total number of features across all 48 target items). If all nine patients are included in a group analysis then significantly more associative than physical information was produced in both picture definition (Wilcoxon $Z=13.0, p<.001$ ) and word definition $(Z=9.57, p<.001)$. This pattern is repeated for each individual patient's results (Wilcoxon $Z$ ranges from 2.68 to 4.47 , all $p<$ .01 ) with the exception of DG for whom there was no significant difference between the number of physical and associative features produced, for either pictures or words.

The final analyses of the patients' definitions assessed any potential differences in performance for concepts related to living things and artifacts. The mean numbers of novel physical and associative attributes produced for the 24 living things and 24 artifacts are illustrated in Tables $4 \mathrm{a}$ and $4 \mathrm{~b}$ for picture and word definitions, respectively. Two ANOVAs revealed significant main effects of attribute type (picture definitions: $F(1,430)=315.8, p<.001$; word definitions: $F(1,430)=113.0, p<.001$ ), semantic category (picture definitions: $F(1,430)=5.3, p=.02$; word definitions: $F(1,430)=9.3$, $p=.002$ ), and feature type $\times$ semantic category interactions (picture definitions: $F(1,430)=62.6, p<.001$; word definitions: $F(1,430)=28.4, p<$ $.001)$. Post-hoc $t$ tests confirm that the interactions arose because a significantly greater number of associative features were produced for artifacts than for living things (picture definition: $t=5.45, p<.001$; word definition: $t=$ $4.84, p<.001$ ), but there was no difference across category for physical attributes produced in word definition $(t<1)$, and the patients gave a smaller number of novel physical features for artifacts in picture definition $(t=6.94$, $p<.001)$.

The influence of semantic category noted in these analyses raises the possi-

${ }^{1}$ Note that in Fig. 2a the bars corresponding to Total are the same figures as Picture: Novel in Fig. 1, likewise the Total bars in Fig. 2b are the same as Word: Total in Fig. 1. 

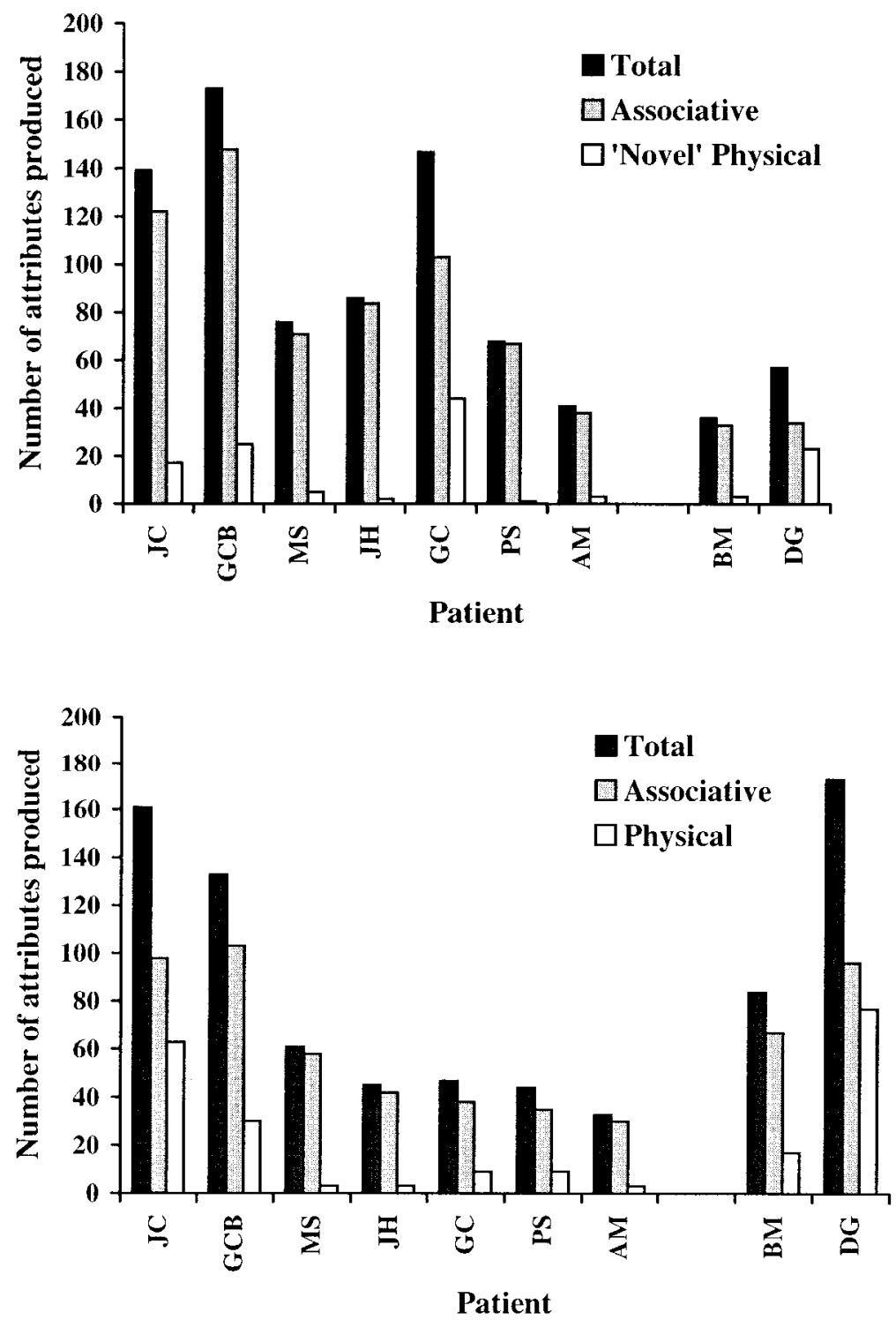

FIG. 2. (a) The number of novel physical and associative features produced in picture definitions. (b) The number of physical and associative features produced in word definitions. 
TABLE 4a

The Mean Number of Features Produced in Each Picture Definition Split by Attribute Type and Semantic Domain

\begin{tabular}{lccr}
\hline & \multicolumn{2}{c}{ Attribute type } \\
\cline { 2 - 3 } Semantic domain & Physical (Novel) & Associative & Overall \\
\hline Living things & $0.49(0.82)$ & $1.23(1.38)$ & $1.72(1.67)$ \\
Artifacts & $0.08(0.29)$ & $2.01(1.58)$ & $2.09(1.63)$ \\
\hline
\end{tabular}

Note. Standard deviations are in parentheses.

bility that some or all of these patients were demonstrating a category-specific deficit for living things. The nine patients did produce more featurerich definitions to artifact concepts than to living things both for words and pictures (see Tables $4 \mathrm{a}$ and $4 \mathrm{~b}$ ). If the number of features produced per item is predicted in a linear regression model by category, then the difference favoring artifacts is significant for both words $(t=3.05, p=.002)$ and pictures $(t=2.30, p=.02)$. We have noted above, however, that the patients' definition performance was significantly modulated by concept familiarity, and there is a significant discrepancy between the two categories on this dimension (mean rated familiarity: artifacts $=3.25$, living things $=2.28$; $t=4.07, p<.001$ ). If rated familiarity and category are included in the simultaneous linear regression model, the patients produced the greatest amount of information for the most familiar concepts both for words ( $t=$ $7.54, p<.001)$ and for pictures $(t=3.63, p<.001)$, but no effect of category remains once familiarity is taken into account (words: $t<1$; pictures: $t=$ -1.15 , n.s.).

In summary of the results presented in this section, although the patients' performances were not significantly affected by semantic category once concept familiarity was taken into account, the clear and striking finding was

TABLE 4b

The Mean Number of Features Produced in Each Word Definition Split by Attribute Type and Semantic Domain

\begin{tabular}{lccc}
\hline & \multicolumn{2}{c}{ Attribute type } & \\
\cline { 2 - 3 } Semantic domain & Physical & Associative & Overall \\
\hline Living things & $0.54(1.07)$ & $0.94(1.41)$ & $1.48(2.11)$ \\
Artifacts & $0.45(1.16)$ & $1.68(1.73)$ & $2.13(2.33)$ \\
\hline
\end{tabular}

Note. Standard deviations are in parentheses. 
that each and every patient produced more (in eight of nine cases, significantly more) associative than physical features in both word and picture definitions. This result implies a substantial degree of functional localization of these two aspects of conceptual knowledge, with the physical attributes of object knowledge particularly reliant on the inferior temporal regions that are compromised in semantic dementia.

\section{GENERAL DISCUSSION}

As described in previous reports of semantic dementia (Hodges et al., 1992; Snowden et al., 1989), the patients in this study presented with a relatively selective progressive semantic impairment resulting in poor comprehension and profound word-finding difficulties. Not surprisingly, the patients with the severest impairment produced the least detailed definitions in response to both words and pictures. Our analyses were designed to assess two key theoretical aspects of semantic organization: first, whether there are separate central semantic subsystems accessed by objects (or in this case, pictures of them) and words and, second, whether there are separate semantic subsystems for different classes of conceptual knowledge, namely, sensory/ physical and functional/associative attributes.

In the Introduction we reviewed some of the evidence taken for and against a modality-specific organization of semantic knowledge. One of the difficulties for research in this area is that a number of key assumptions underlying this view are still underspecified. Deriving an exact set of empirical predictions is, consequently, rather difficult. One approach to this problem is to treat the amodal single semantic hypothesis as the default, because it is the more parsimonious, theoretical position, and to ask whether there is any evidence that compels us to accept more than one system for semantic representations of concrete concepts.

The comparison of definitions in response to words and pictures for the nine patients presented here seems, in many ways, to reflect the existing claims in the literature for separate verbal and visual semantic systems. All these patients make confusions between exemplars drawn from the same category in comprehension tasks like word-to-picture matching and make frank semantic errors in naming. Such data provide converging evidence for a semantic locus to the functional impairment in all nine cases. Some of the patients (four out of nine) produced significantly better definitions to pictures than to words and likewise achieved higher scores in comprehension tasks like the Pyramids and Palm Trees test with picture stimuli than with word stimuli. Two other patients exhibited the reverse pattern. Consequently, at this level of analysis the data seem to reinforce the apparent double dissociation formed by individual cases reported in the literature, e.g., patients RM (Lauro-Grotto et al., 1997) vs. FRA and DRS (McCarthy \& Warrington, 1986; Warrington \& McCarthy, 1994). 
The point that we wish to emphasize is that this double dissociation in performance does not necessarily demonstrate multiple semantic systems. In fact, as we intimated in the Introduction, the evidence taken as favoring the modality-specific hypothesis has legitimate alternative interpretations. First, it is important to note that, although some of the patients exhibited a degree of differential word/picture performance, in no case could this be considered to approach a classical dissociation (Shallice, 1988a). That is, all nine subjects displayed impaired comprehension of both pictures and words. This means that, with a modality-specific theory, we would need to postulate damage to both semantic systems albeit to different degrees. The amodal theory, on the other hand, predicts an impact of a semantic deficit on comprehension in all modalities. The fact that many patients achieve poorer scores on tests of word relative to picture comprehension could result from the fact that the surface form of a word has an arbitrary relationship with its meaning whereas the meaning of an object is signaled substantially more by features present in the stimulus. The systematicity in the mapping, or the affordance, from objects or pictures to semantics means that comprehension of these should be relatively resistant to disruption.

This notion of an inherent bias toward an advantage for objects or pictures requires us to explain how there can be any patients who present with relatively better comprehension of words than of pictures (patients BM and DG in the present study. Our response is that it is not clear that the less-detailed definitions produced by BM and DG for pictures than for words do constitute evidence for a dissociation within central conceptual knowledge. Although there is no doubt that both patients had a central semantic impairment, both also performed poorly and well below both the normal range and the other patients with semantic dementia on simple assessments of presemantic perceptual processing (e.g., matching the same object across two different views). Given a combination of impaired conceptual knowledge and a visuoperceptual deficit, it is unsurprising that these patients produce relatively poor definitions of picture input and are disproportionately impaired on other tests of picture comprehension.

In addition to the fact that a single amodal semantic system may sensibly be considered the theoretical default, we have reported some data in this study that seem to argue positively in favor of this hypothesis. In line with the results of three previous studies of the semantic impairment in dementia of Alzheimer's type (Chertkow et al., 1992; Hodges et al., 1996; Lambon Ralph et al., 1997), we identified significant associations between word and picture definitions and between picture naming and word definitions. Furthermore, these reliable associations were not explicable by the influence of additional factors such as concept familiarity on the patients' performance. The associations indicate that the status of conceptual information when probed from verbal input (the concept name) is a good predictor of the quality of 
semantic knowledge when accessed from pictorial input, either for comprehension or for naming. ${ }^{2}$

The functional imaging study reported by Vandenberghe et al. (1996) also points toward a common distributed semantic system for words and pictures. In addition, however, these authors reported increased regional cerebral blood flow (rCBF) specifically for word comprehension in the left superior temporal sulcus, left anterior middle temporal gyrus, and left inferior frontal sulcus and for the picture semantic tasks in the left posterior inferior temporal sulcus. Vandenberghe et al. suggested that $\mathrm{rCBF}$ increases in these regions may be attributable either to activation of modality-specific input representations (structural descriptions in the case of pictures, form-based representations for words) or to the transmission of activation from these input representations to shared conceptual knowledge. The results from this functional imaging study are consistent with the view proposed here, in which we attribute the extra-poor definitions provided by DG and BM for pictures to the additional presemantic visuo-perceptual deficits observed in these two patients. Our assessment is that there is currently no empirical evidence, either from studies of behavioral deficits in patients or from functional imaging of intact subjects, that necessitates a modality-specific viewpoint. If anything, the item consistency noted in this and previous studies would seem to favor the single, amodal hypothesis.

Semantic dementia arises from progressive atrophy of the inferior and lateral aspects of the temporal lobes (Hodges \& Patterson, 1996; Hodges et al., 1992; Mummery et al., 1999), areas assumed to underpin knowledge about the physical form of objects and animals (Ungerleider \& Mishkin, 1982). This anatomical specificity would suggest that, on top of their generalized semantic degradation, these patients should exhibit disproportionate loss of conceptual knowledge with respect to this specific type of semantic feature. We noted in the Introduction that previously studied patients had demonstrated this effect across a variety of tasks (Basso et al., 1988; Breedin et al., 1994b; Cardebat et al., 1996; Lambon Ralph et al., 1998b; Moss et al., 1995; Tyler \& Moss, 1997). The analyses of the definitions provided by the nine patients included in this study replicate these previous findings: whether

\footnotetext{
${ }^{2}$ The problem of resolving empirical predictions from the modality-specific hypothesis is highlighted here. If we consider the verbal and visual semantics as distinct, independent entities, then there would be no reason to expect that damage to one or both modalities should produce the type of item consistency that has been observed here (i.e., consistency greater than that expected on the basis of concept familiarity and other variables). If the two systems are linked, or coupled (Shallice, 1988a), in some way, such that activation can pass between corresponding semantic representations, then the prediction is less than clear. In the limit, damage affecting a representation in one modality may make it less likely for the corresponding concept to be activated in the other. At a functional level of description, therefore, a highly interactive modality-specific view of this sort may well behave in exactly the same way as an impaired amodal semantic system.
} 
defining pictures or words, all patients produced strikingly less physical/ sensory than associative/functional information. This pattern is not true of normal control subjects who, if anything, produce more physical than associative information (Hodges et al., 1996). In some cases the patients' definitions contained almost no physical/sensory attributes. These results, as well as data from a recent PET imaging study of normal subjects (Cappa, Perani, Schmur, Tettamanti, \& Fazio, 1998; Mummery, Patterson, Hodges, \& Price, 1998), support models of semantic memory in which distributed conceptual representations are composed of subsets of sensory and associative/functional attributes based on partially different cortical regions (e.g., Allport, 1985; Lambon Ralph et al., 1997; Saffran \& Sholl, in press; Warrington \& McCarthy, 1987).

A popular hypothesis of category structure in semantic memory views living things as primarily differentiated on the basis of sensory features, while function is thought to be the key distinguishing aspect of artifacts (Farah \& McClelland, 1991; Lambon Ralph et al., 1997; Saffran \& Schwartz, 1994; Saffran \& Sholl, in press; Warrington \& Shallice, 1984). Farah and McClelland demonstrated that, if this asymmetric weighting of sensory and functional features were encoded within a connectionist model of semantic memory, then apparent category-specific deficits for living things would arise from a selective 'lesion' of sensory attributes. Given the apparently sparse knowledge of patients with semantic dementia about the physical form of objects and animals, one might predict that the great majority of these patients should exhibit disproportionately poor performance on living things. Category-specific deficits for natural kinds have been reported for a few individual patients with semantic dementia (Barbarotto, Capitani, Spinnler, \& Trivelli, 1995; Basso et al., 1988; Breedin, Martin, \& Saffran, 1994a; Cardebat et al., 1996; McCarthy \& Warrington, 1988); but with the exception of the study by Barbarotto et al., it is unclear whether these effects could be explained by a difference between categories on factors such as familiarity and frequency which are known to have a significant impact on performance (Funnell, 1995; Lambon Ralph et al., 1998a). Patient MF, reported by Barbarotto et al. (1995), did demonstrate a significant category-specific effect after various other pertinent factors were taken into account; conversely, MF did not exhibit a significant difference between sensory and associative knowledge - a pattern also reported in several other recent papers on category-specific deficits (Caramazza \& Shelton, 1998; Funnell \& De Mornay Davies, 1996; Laiacona, Barbarotto, \& Capitani, 1993; Laiacona, Capitani, \& Barbarotto, 1997; Lambon Ralph et al., 1998b; Moss, Tyler, Durrant-Peatfield, \& Bunn, 1998; Samson, De Wilde, \& Pillon, 1998).

Do the patients described here exhibit significant category specific effects? There are three sets of relevant results. Analyses of the definition data from the present study revealed that the patients as a group did produce richer definitions for artifact concepts than for living things both to words and pic- 
tures. The patients' definition performance was also significantly modulated by concept familiarity, however, and when this factor is taken into account, no category effect remains. Hodges, Garrard, and Patterson (1998) analyzed data from the word-to-picture matching and naming tasks (Hodges \& Patterson, 1996) for a set of eight patients, including a number of the cases reported here. With rated familiarity controlled, there was a significant advantage for artifacts relative to natural kinds in the word-to-picture matching task, but no effect of category type on naming to confrontation. Six of the patients were also included in a case-series study of the factors that affect naming accuracy for a set of 132 pictures from the Snodgrass and Vanderwart corpus (Lambon Ralph et al., 1998a). As a group there was no effect of category on naming accuracy and in the individual analyses only one patient exhibited significantly better performance for the artifacts.

In summary, the weight of evidence appears not to favor models of semantic memory in which category-specific deficits arise from a differential weighting of sensory and functional features for living kinds and artifacts. On the other hand it is, of course, difficult to draw firm conclusions from a set of null results. All the patients reported here produced impoverished definitions overall, and although they gave strikingly little sensory/physical information, they also only tended to produce, on average, between one and three pieces of associative information per item (see Figs. 2a and 2b). With such a severe reduction in both physical and associative aspects of conceptual knowledge, one might not expect a measurable category effect.

\section{CONCLUSIONS}

Analysis of word and picture definitions given by nine patients with semantic dementia revealed (a) that the quality of the information produced by the patients declined with disease severity, (b) that more satisfactory (though still impoverished) definitions were given to the most familiar concepts, and (c) that once concept familiarity was taken into account the patients' performance was equivalent for artifacts and living things. With regard to the specific theoretical questions to which the study was addressed, we found a striking reduction in the physical attributes of conceptual knowledge produced by all nine patients to both words and pictures. In addition, the ability to give a core definition to a specific word was highly predictive of the patients' success in both picture comprehension and confrontation naming for the same item, suggesting impairment of a single amodal semantic system. The relatively poorer definitions to pictures than to words produced by two of the nine patients was explicable by their combined conceptual and presemantic, visuo-perceptual deficits. It is our contention, therefore, that there is no evidence from this study (or perhaps in the literature as a whole) that necessitates a modality-specific semantic organization. Rather than positing impairment to a separate conceptual system for verbal compre- 
hension as an explanation for the relatively common pattern of poorer performance with words than with pictures, we would argue that this pattern arises from the underlying difference in mapping to meaning that makes a picture worth a thousand words.

\section{REFERENCES}

Allport, D. A. 1985. Distributed memory, modular subsystems and dysphasia. In S. Newman \& R. Epstein (Eds.), Current perspectives in dysphasia. Edinburgh: Churchill Livingstone. Baayen, R. H., Piepenbrock, R., \& van Rijn, H. 1993. The CELEX lexical database [CDROM]. Philadelphia, PA: Linguistics Data Consortium, Univ. of Pennsylvania.

Barbarotto, R., Capitani, E., Spinnler, H., \& Trivelli, C. 1995. Slowly progressive semantic impairment with category specificity. Neurocase, 1, 107-119.

Basso, A., Capitani, E., \& Laiacona, M. 1988. Progressive language impairment without dementia: A case with isolated category specific semantic defect. Journal of Neurology, Neurosurgery and Psychiatry, 51, 1201-1207.

Behrmann, M., \& Bub, D. 1992. Surface dyslexia and dysgraphia: Dual routes, single lexicon. Cognitive Neuropsychology, 9, 209-251.

Benton, A. L., Hamsher, K., Varney, N., \& Spreen, O. 1983. Contributions to neuropsychological assessment. Oxford: Oxford Univ. Press.

Breedin, S. D., Martin, N., \& Saffran, E. M. 1994a. Category-specific semantic impairments: An infrequent occurrence. Brain and Language, 47, 383-386.

Breedin, S. D., Saffran, E. M., \& Coslett, H. B. 1994b. Reversal of the concreteness effect in a patient with semantic dementia. Cognitive Neuropsychology, 11, 617-660.

Bub, D., Black, S., Hampson, E., \& Kertesz, A. 1988. Semantic encoding of pictures and words: Some neuropsychological observations. Cognitive Neuropsychology, 5, 27-66.

Cappa, S. F., Perani, D., Schmur, T., Tettamanti, M., \& Fazio, F. 1998. The effects of semantic category and knowledge type on lexical-semantic access: A PET study. Neuroimage, $\mathbf{8}$, $350-359$.

Caramazza, A., Hills, A. E., Rapp, B. C., \& Romani, C. 1990. Multiple semantics or multiple confusions? Cognitive Neuropsychology, 7, 161-168.

Caramazza, A., \& Shelton, J. R. 1998. Domain-specific knowledge systems in the brain: The animate-inanimate distinction. Journal of Cognitive Neuroscience, 10, 1-34.

Carbonnel, S., Charnallet, A., David, D., \& Pellat, J. 1997. One or several semantic systems(s)? Maybe none: Evidence from a case of modality and category-specific "semantic" impairment. Cortex, 33, 391-417.

Cardebat, D., Demonet, J. F., Celsis, P., \& Puel, M. 1996. Living/non-living dissociation in a case of semantic dementia: A SPECT activation study. Neuropsychologia, 34, 11751179.

Chertkow, H., Bub, D., \& Caplan, D. 1992. Constraining theories of semantic memory processing: Evidence from dementia. Cognitive Neuropsychology, 9, 327-365.

Coltheart, M. 1981. The MRC psycholinguistic database. Quarterly Journal of Experimental Psychology, 33, 497-505.

Coltheart, M., \& Funnell, E. 1987. Reading and writing: One lexicon or two? In D. A. Allport, D. G. MacKay, W. Prinz, \& E. Scheerer (Eds.), Language perception and production: Shared mechanisms in listening, reading and writing. London: Academic Press.

Coltheart, M., Inglis, L., Cupples, L., Michie, P., Bates, A., \& Budd, B. 1998. A semantic 
subsystem specific to the storage of information about visual attributes of animate and inanimate objects. Neurocase, 4, 353-370.

Farah, M. J., \& McClelland, J. L. 1991. A computational model of semantic memory impairment: Modality specificity and emergent category specificity. Journal of Experimental Psychology General, 120, 339-357.

Funnell, E. 1995. Objects and properties: A study of the breakdown of semantic memory. Memory, 3, 497-518.

Funnell, E., \& De Mornay Davies, P. 1996. JBR: A reassessment of concept familiarity and a category-specific disorder for living things. Neurocase, 2, 461-474.

Gibson, J. J. 1979. The ecological approach to visual perception. Boston: Houghton Mifflin.

Graham, K. S., Becker, J. T., Patterson, K., \& Hodges, J. R. 1997a. Lost for words: A case of primary progressive aphasia? In A. J. Parkin (Ed.), Case studies in the neuropsychology of memory. Hove: Erlbaum.

Graham, K. S., \& Hodges, J. R. 1997. Differentiating the roles of the hippocampal complex and the neocortex in long-term memory storage: Evidence from the study of semantic dementia and Alzheimer's disease. Neuropsychology, 11, 77-89.

Graham, K. S., Hodges, J. R., \& Patterson, K. E. 1994. The relationship between comprehension and oral reading in progressive fluent aphasia. Neuropsychologia, 32, 299-316.

Graham, K. S., Lambon Ralph, M. A., \& Hodges, J. R. 1997b. Determining the impact of autobiographical experience on 'meaning': New insights from investigating sports-related vocabulary and knowledge in two cases with semantic dementia. Cognitive Neuropsychology, 14, 801-837.

Graham, K. S., Murre, J. M. J., \& Hodges, J. R. in press. Episodic memory in semantic dementia: A computational approach based on the TraceLink model. In J. Reggia, E. Ruppin, \& D. Glanzman (Eds.), Neural modelling in brain disorders. Oxford: Elsevier.

Hillis, A. E., Rapp, B., \& Caramazza, A. 1995. Constraining claims about theories of semantic memory: More on unitary versus multiple semantics. Cognitive Neuropsychology, 12, 175-186.

Hodges, J. R., Garrard, P., \& Patterson, K. 1998. Semantic dementia. In A. Kertesz \& D. G. Munoz (Eds.), Pick's disease and Pick complex. New York: Wylie-Liss.

Hodges, J. R., \& Graham, K. S. 1998. A reversal of the temporal gradient for famous person knowledge in semantic dementia: Implications for the neural organisation of long-term memory. Neuropsychologia, 36, 803-825.

Hodges, J. R., \& Patterson, K. 1995. Is semantic memory consistently impaired early in the course of Alzheimer's disease? Neuroanatomical and diagnostic implications. Neuropsychologia, 33, 441-459.

Hodges, J. R., \& Patterson, K. 1996. Nonfluent progressive aphasia and semantic dementia: A comparative neuropsychological study. Journal of the International Neuropsychological Society, 2, 511-524.

Hodges, J. R., Patterson, K., Graham, N., \& Dawson, K. 1996. Naming and knowing in dementia of Alzheimer's type. Brain and Language, 54, 302-325.

Hodges, J. R., Patterson, K., Oxbury, S., \& Funnell, E. 1992. Semantic dementia: Progressive fluent aphasia with temporal lobe atrophy. Brain, 115, 1783-1806.

Hodges, J. R., Patterson, K., \& Tyler, L. K. 1994. Loss of semantic memory: Implications for the modularity of mind. Cognitive Neuropsychology, 11, 505-542.

Howard, D. 1995. Lexical anomia: Or the case of the missing lexical entries. Quarterly Journal of Experimental Psychology Human Experimental Psychology, 48a, 999-1023.

Howard, D., \& Patterson, K. 1992. The Pyramids and Palm Trees Test: A test of semantic access from words and pictures. Bury St. Edmunds: Thames Valley Test Company. 
Knott, R., Patterson, K., \& Hodges, J. R. 1997. Lexical and semantic binding effects in shortterm memory: Evidence from semantic memory Cognitive Neuropsychology, 8, 11651216.

Laiacona, M., Barbarotto, R., \& Capitani, E. 1993. Perceptual and associative knowledge in category specific impairment of semantic memory: A study of two cases. Cortex, 29, 727-740.

Laiacona, M., Capitani, E., \& Barbarotto, R. 1997. Semantic category dissociations: A longitudinal study of two cases. Cortex, 33, 441-461.

Lambon Ralph, M. A., Graham, K. S., Ellis, A. W., \& Hodges, J. R. 1998a. Naming in semantic dementia-What matters? Neuropsychologia, 36, 775-784.

Lambon Ralph, M. A., Howard, D., Nightingale, G., \& Ellis, A. W. 1998b. Are living and non-living category-specific deficits causally linked to impaired perceptual or associative knowledge? Evidence from a category-specific double dissociation. Neurocase, 4, 311338.

Lambon Ralph, M. A., Patterson, K., \& Hodges, J. R. 1997. The relationship between naming and semantic knowledge for different categories in dementia of Alzheimer's type. Neuropsychologia, 35, 1251-1260.

Lauro-Grotto, R., Piccini, C., \& Shallice, T. 1997. Modality-specific operations in semantic dementia. Cortex, 33, 593-622.

Marshall, J., Pring, T., Chiat, S., \& Robson, J. 1996. Calling a salad a federation: An investigation of semantic jargon. Part 1-Nouns. Journal of Neurolinguistics, 9, 237-250.

McCarthy, R. A., \& Warrington, E. K. 1986. Visual associative agnosia: A clinico-anatomical study of a single case. Journal of Neurology, Neurosurgery and Psychiatry, 49, 12331240 .

McCarthy, R. A., \& Warrington, E. K. 1988. Evidence for modality-specific meaning systems in the brain. Nature, 334, 428-430.

McKenna, P., \& Warrington, E. K. 1980. A test for nominal dysphasia. Journal of Neurology, Neurosurgery and Psychiatry, 43, 781-788.

Milner, A. D., \& Goodale, M. A. 1995. The visual brain in action. Oxford: Oxford Univ. Press.

Morton, J. 1985. Naming. In S. Newman \& R. Epstein (Eds.), Current perspectives in dysphasia. Edinburgh: Churchill Livingstone.

Moss, H. E., Tyler, L. K., Durrant-Peatfield, M., \& Bunn, E. M. 1998. Two eyes of a seethrough: Impaired and intact semantic knowledge in a case of selective deficit for living things. Neurocase, 4, 291-310.

Moss, H. E., Tyler, L. K., Hodges, J. R., \& Patterson, K. 1995. Exploring the loss of semantic memory in semantic dementia: Evidence from a primed monitoring study. Neuropsychology, 9, 16-26.

Mummery, C. J., Patterson, K., Hodges, J. R., \& Price, C. J. 1998. Functional neuroanatomy of the semantic system: Divisible by what? Journal of Cognitive Neuroscience, 10, 766777.

Mummery, C. J., Patterson, K., Wise, R. J. S., Price, C. J., \& Hodges, J. R. 1999. Disrupted temporal lobe connections in semantic dementia. Brain, 122, 61-73.

Osterrieth, P. 1944. Le test de copie d'une figure complexe. Archives de Psychologie, 30, 205-550.

Plaut, D. C., McClelland, J. L., Seidenberg, M. S., \& Patterson, K. 1996. Understanding normal and impaired word reading: Computational principles in quasi-regular domains. Psychological Review, 103, 56-115. 
Plaut, D. C., \& Shallice, T. 1993. Deep dyslexia: A case study of connectionist neuropsychology. Cognitive Neuropsychology, 10, 377-500.

Rapp, B. C., Hillis, A. E., \& Caramazza, A. 1993. The role of representations in cognitive theory: More on multiple semantics and the agnosias. Cognitive Neuropsychology, 10, 235-249.

Raven, J. C. 1962. Coloured progressive matrices: Sets A, AB, B. London: HK Lewis.

Riddoch, M. J., \& Humphreys, G. W. 1992. The Birmingham Object Recognition Battery (BORB). Hove: Erlbaum.

Riddoch, M. J., Humphreys, G. W., Coltheart, M., \& Funnell, E. 1988. Semantic systems or system? Neuropsychological evidence re-examined. Cognitive Neuropsychology, 5, 325.

Saffran, E. M., \& Schwartz, M. F. 1994. Of cabbages and things: Semantic memory from a neuropsychological perspective-A tutorial review. Attention and Performance, 25, 507536.

Saffran, E. M., \& Sholl, A. in press. Clues to the functional and neural architecture of word meaning. In P. Hagoort \& C. Brown (Eds.), Neurocognition of language. Oxford: Oxford Univ. Press.

Samson, D., De Wilde, V., \& Pillon, A. 1998. Impaired knowledge of visual and nonvisual attributes in a patient with a naming impairment for living entities: A case of a true category-specific deficit. Neurocase, 4, 273-290.

Schwartz, M. F., Marin, O. S. M., \& Saffran, E. M. 1979. Dissociations of language function in dementia: A case study. Brain and Language, 7, 277-306.

Shallice, T. 1988a. From neuropsychology to mental structure. Cambridge: Cambridge Univ. Press.

Shallice, T. 1988b. Specialisation within the semantic system. Cognitive Neuropsychology, 5, $133-142$.

Shallice, T. 1993. Multiple semantics: Whose confusions? Cognitive Neuropsychology, 10, 251-261.

Sirigu, A., Duhamel, J.-R., \& Poncet, M. 1991. The role of sensorimotor experience in object recognition. Brain, 1991, 2555-2573.

Snodgrass, J. G., \& Vanderwart, M. 1980. A standardised set of 260 pictures: Norms for name agreement, image agreement, familiarity, and visual complexity. Journal of Experimental Psychology: Human Learning and Memory, 6, 174-215.

Snowden, J. S., Goulding, P. J., \& Neary, D. 1989. Semantic dementia: A form of circumscribed cerebral atrophy. Behavioural Neurology, 2, 167-182.

Srinivas, K., Breedin, S. D., Coslett, H. B., \& Saffran, E. M. 1997. Intact perceptual priming in a patient with damage to the anterior inferior temporal lobes. Journal of Cognitive Neuroscience, 9, 490-511.

Tanabe, H., Nakagawa, Y., Ikeda, M., Hashimoto, M., Yamada, N., Kazui, H., Nishikawa, T., \& Okuda, J. 1996. Selective loss of semantic memory for words. In K. Ishikawa, J. L. McGaugh, \& H. Sakata (Eds.), Brain Processes and Memory. B. V.: Elsevier.

Tyler, L. K., \& Moss, H. E. 1997. Functional properties of concepts: Studies of normal and brain-damaged patients. Cognitive Neuropsychology, 14, 511-545.

Tyler, L. K., \& Moss, H. E. 1998. Going, going, gone? . . . Implicit and explicit tests of conceptual knowledge in longitudinal study of semantic dementia. Neuropsychologia, 36, 1313-1323.

Ungerleider, L. G., \& Mishkin, M. 1982. Two cortical visual systems. In D. J. Ingle, M. A. Goodale, \& R. J. W. Mansfield (Eds.), Analysis of visual behaviour. Cambridge, MA: MIT Press. 
Vandenberghe, R., Price, C., Wise, R., Josephs, O., \& Frackowiak, R. S. J. 1996. Functionalanatomy of a common semantic system for words and pictures. Nature, 383, 254-256.

Warrington, E. K. 1975. The selective impairment of semantic memory. Quarterly Journal of Experimental Psychology, 27, 635-657.

Warrington, E. K., \& McCarthy, R. 1987. Categories of knowledge: Further fractionations and an attempted integration. Brain, 110, 1273-1296.

Warrington, E. K., \& McCarthy, R. A. 1994. Multiple meaning systems in the brain: A case for visual semantics. Neuropsychologia, 32, 1465-1473.

Warrington, E. K., \& Shallice, T. 1984. Category specific semantic impairments. Brain, 107, 829-854. 